\title{
Fatores que impedem a adequação da oferta nutricional enteral em pacientes críticos
}

\author{
Factors that hinder the adequacy of enteral nutritional supply in critically ill patients
}

\section{DOI: 10.37111/braspenj.2019344004}

Mayra da Rosa Martins Walczewski

Eduardo André Bracci Walczewski

Carolina Exterkotter Wiggers ${ }^{3}$

Beatriz Camilo da Rosa ${ }^{3}$

Francisco Henrique Cavalli Marconato ${ }^{4}$

\section{Unitermos:}

Nutrição enteral. Unidades de terapia intensiva. Terapia nutricional.

\section{Keywords:}

Enteral nutrition. Intensive care units. Nutrition therapy.

\section{Endereço para correspondência:}

Mayra da Rosa Martins Walczewski

Rua Dona Antonina Búrigo Corbetta, 119 - Vila Moe-

ma - Tubarão - SC, Brasil - CEP 88705-030

E-mail: mayrarmartins@hotmail.com

\section{Submissão}

17 de outubro de 2019

\section{Aceito para publicação}

7 de novembro de 2019

\begin{abstract}
RESUMO
Introdução: A desnutrição calórico proteica é prevalente nos pacientes críticos. Isso pode ser agravado com uma oferta inadequada de nutrição enteral. Logo, é crucial investigar a adequação nutricional e identificar as barreiras mais frequentes à sua administração. Método: Estudo longitudinal realizado entre junho e dezembro de 2017, na unidade de terapia intensiva (UTI) de um hospital do sul de Santa Catarina, aprovado pelo CEP, sob o protocolo número 2.274.728. Foram incluídos pacientes $\geq 18$ anos em terapia de nutrição enteral (TNE) por um período $\geq 72$ horas. Foram coletados dados sociodemográficos, dados referentes à nutrição enteral e à internação na UTI e exames laboratoriais. Resultados: Dentre os pacientes internados no setor, 147 foram avaliados. Destes, $78,9 \%$ receberam $\geq 70 \%$ da dieta prescrita e $36,1 \%(n=53)$ iniciaram a TNE apenas após a admissão na UTI, com mediana de início de 20 (13-33) horas. O principal motivo de interrupção da TNE foi jejum para procedimento em $37,4 \%(n=55)$ dos pacientes analisados. A inadequação nutricional foi associada à presença de vômito $(p=0,012)$ e distensão abdominal $(p=0,009)$, assim como à menor duração da TNE $(p=0,017)$ e ao maior tempo para início da TNE $(p=0,046)$. Conclusão: $O$ início precoce da TNE está de acordo com o preconizado pela diretriz. $A$ barreira à oferta nutricional mais prevalente foi jejum para procedimentos. A presença de alguns sintomas gastrointestinais, como vômitos e distensão abdominal, menor tempo de TNE e maior tempo para início dessa parecem estar associados à inadequação calórico proteica.
\end{abstract}

\section{ABSTRACT}

Introduction: Caloric protein malnutrition is prevalent in critically ill patients. This can be aggravated by an inadequate supply of enteral nutrition. Therefore, it is crucial to investigate nutritional adequacy and to identify the most frequent barriers to its administration. Methods: A longitudinal study was performed between June and December 2017 at the intensive care unit (ICU) of a hospital in the South of Santa Catarina, approved under the protocol 2.274.728. Patients $\geq 18$ years, in enteral nutrition therapy (ENT) for $\geq 72$ hours were included. Socio-demographic data, ICU length of stay and nutrition therapy and laboratory tests were collected. Results: Among the patients in the unit, 147 were evaluated. Of these, $78.9 \%$ received $\geq 70 \%$ of the prescribed diet and $36.1 \%(n=53)$ started the EN only after ICU admission, with median onset of 20 (13-33) hours. The main reason for interruption of NET was fasting for procedures in $37.4 \%(n=55)$ of the patients analyzed. Nutritional inadequacy was associated with the presence of vomiting $(p=0.012)$ and abdominal distension $(p=0,009)$, such as shorter use of ENT $(p=0.017)$ and longer time to ENT $(p=0,046)$. Conclusions: The early start of the ENT is in line with what is recommended by the guidelines. The most prevalent nutritional barrier was fasting for procedures. The presence of some gastrointestinal symptoms, such as vomiting and abdominal distention, shorter time to ENT and longer time to start of ENT appear to be associated with protein calorie inadequacy.

1. Médica gastroenterologista e coordenadora clínica da EMTN no Hospital Nossa Senhora da Conceição em Tubarão - SC e professora da disciplina Sistema Digestivo na Universidade do Sul de Santa Catarina (UNISUL), campus Tubarão, SC, Brasil.

2. Médico cirurgião e gastroenterologista no Hospital Nossa Senhora da Conceição em Tubarão - SC e professor da disciplina Sistema Digestivo na Universidade do Sul de Santa Catarina (UNISUL), campus Tubarão - SC, Brasil.

3. Acadêmica de medicina na Universidade do Sul de Santa Catarina (UNISUL), campus Tubarão - SC, Brasil.

4. Médico residente no serviço de cirurgia geral no Hospital Nossa Senhora da Conceição em Tubarão - SC, Brasil. 


\section{INTRODUÇÃO}

A depleção nutricional é frequente e um dos maiores problemas em pacientes hospitalizados em unidades de terapia intensiva (UTI) ${ }^{1,2}$. Há uma prevalência de desnutrição de cerca de $50 \%$ nos internados na UTI, sendo que mais de $35 \%$ dos pacientes já são admitidos nessa condição ${ }^{3}$.

Apesar de causar prejuízo à saúde dos internados, a má nutrição é muitas vezes negligenciada e apresenta algumas complicações que resultam no aumento da morbimortalidade e prolongamento da internação. São exemplos: resposta imunológica reduzida, comprometimento do processo de cicatrização, alterações na composição corporal e na função dos órgãos, além de maior susceptibilidade às infecções ${ }^{2,4}$. Tais consequências podem ser atenuadas pelo tratamento com uma terapia nutricional adequada ${ }^{4}$.

A terapia de nutrição enteral (TNE) é a primeira opção quando a alimentação não for possível por via oral, desde que não haja contraindicação absoluta a sua utilização. $\bigcirc$ início precoce da TNE, de 24 a 48 horas após admissão, é recomendado, sendo que o alcance das necessidades energéticas deverá acontecer em 48 a 72 horas $^{5}$.

As evidências sugerem que o uso da TNE é capaz de atender à demanda dos nutrientes necessários, além de manter a integridade intestinal, atenuar a resposta inflamatória, diminuir a translocação bacteriana, reduzir o tempo de internação e risco de infecções e complicações e impacta favoravelmente o desfecho dos pacientes. Logo, analisar a eficácia desse tratamento é fundamental $\left.\right|^{6,7}$.

Apesar dos vários benefícios do uso da nutrição enteral, nem todos os pacientes admitidos na UTI recebem toda a nutrição prescrita, visto que há diversas barreiras que levam à interrupção da oferta nutricional planejada. Dentre essas intercorrências estão o atraso na administração da dieta, as disfunções gastrointestinais, os problemas relacionados à sonda nasoenteral (SNE), o jejum para a realização de procedimentos e exames e a instabilidade clínica do paciente ${ }^{3,8}$.

Essas interrupções somadas a uma oferta nutricional inadequada podem contribuir significativamente para a incidência de desnutrição calórico proteica em internados na UTI. Por isso, essa adequação representa um grande desafio no tratamento do paciente crítico?

É irreal assumir que todas as interrupções da dieta possam ser impedidas, entretanto, é crucial identificar as barreiras mais frequentes e as mais relacionadas à inadequação nutricional e tentar minimizá-las. Portanto, o objetivo deste estudo consiste em avaliar a adequação de volume e proteína da TNE e identificar os principais fatores que impedem a adequação nutricional em pacientes internados em uma UTI em um hospital do sul de Santa Catarina.

\section{MÉTODO}

Trata-se de uma pesquisa transversal, realizada no período de junho a dezembro de 2017, na UTI do Hospital Nossa Senhora da Conceição (HNSC), em Tubarão, Santa Catarina.
Oestudo foi aprovado pelo Comitê de Ética em Pesquisa da instituição, sob o protocolo de número 2.274.728, no dia 14 de setembro de 2017, e obedeceu aos preceitos da Resolução $466 / 2012$. Para o componente prospectivo da pesquisa foi utilizado o Termo de Consentimento Livre e Esclarecido.

A população foi constituída por 147 pacientes internados na UTI, com idade igual ou superior a 18 anos, de ambos os sexos, que fizeram uso de TNE exclusiva ou associada à via oral por um período maior que 72 horas. Consideraram-se critérios de exclusão pacientes cirúrgicos e vítimas de trauma.

A coleta de dados foi realizada no sistema de prontuários eletrônicos TASY e no software Total Care Danone, em um único momento, após o desfecho do paciente (alta da UTI, suspensão da TNE ou morte), por meio de um protocolo de pesquisa contendo as seguintes variáveis: gênero, idade, peso, altura, índice de massa corporal (IMC), diagnóstico de internação, tempo de internação na UTI, tempo em uso da TNE, número de horas para início da TNE, volume de dieta e proteína prescritos e infundidos, fatores de interrupção da oferta nutricional e proteína C-reativa (PCR) na admissão no setor.

As necessidades energéticas foram estimadas individualmente, conforme Serviço de Nutrição da instituição e diretrizes da Sociedade Americana de Nutrição Parenteral e Enteral ${ }^{5}$. Utilizou-se, para o cálculo da adequação energética, 20-30 $\mathrm{kcal} / \mathrm{kg} /$ dia, sem ajuste para proteína. Porém, para os IMCs maiores que $30 \mathrm{~kg} / \mathrm{m}^{2}$, utilizou-se 11 - $15 \mathrm{kcal} / \mathrm{kg} / \mathrm{dia}$.

Para cada paciente avaliado, foram verificados, a partir do quarto dia de dieta enteral até o desfecho, o volume e a proteína efetivamente ofertados em relação às reais necessidades estimadas. Esse período foi determinado por ser o tempo necessário para a progressão do volume e alcance das necessidades nutricionais. Para a descrição e análise dos resultados, os pacientes foram classificados em 2 grupos: alimentação padrão ( $\geq 70 \%$ de dieta prescrita infundida) ou oferta inadequada de dieta $(<70 \%)^{10}$.

Considerou-se causa de interrupção da oferta nutricional: jejum para procedimentos e exames, problemas relacionados à SNE (saída/obstrução da sonda, demora para troca da via), atraso para liberação da dieta, sintomas gastrointestinais, hemodiálise e outros. Várias interrupções podem ser vistas em um mesmo paciente, por isso, a descrição dos resultados foi em relação ao número de pacientes que apresentaram cada uma delas.

banco de dados foi criado no Microsoft Excel e analisado por meio do programa SPSS 20.0. Para apresentação dos dados, as variáveis categóricas foram expressas em valor absoluto ou porcentagem (\%) e variáveis contínuas em mediana (p25-p75) ou média \pm desvio padrão, conforme indicado. As variáveis categóricas foram analisadas pelo teste de Fisher e qui-quadrado $\left(X^{2}\right)$ e as variáveis numéricas pelo teste Anova. Os testes associaram as variáveis e a inadequação da oferta nutricional ( $<70 \%$ de dieta infundida). A análise foi feita por meio de regressão logística, por isso a medida de associação utilizada foi razão de chances ou odds ratio. $\bigcirc$ nível de significância estatística adotado foi de 5\% (valor de $p<0,05$ ). 


\section{RESULTADOS}

Foram analisados 147 pacientes em uso de TNE na UTI do HNSC internados no período da pesquisa, destes $79(53,7 \%)$ eram do sexo feminino. A idade dos participantes variou de 18 a 95 anos, com mediana de $71(62,0-79,5)$ anos. A média do IMC foi 27,24 55,98. Os dados demográficos e as características clínicas dos pacientes estão apresentados na Tabela 1 .

Os diagnósticos de admissão na UTI mais frequentes foram devido às causas respiratórias ( $n=42)$ e à sepse $(n=39)$. Em outros pacientes $(n=10)$, foram encontrados intoxicações exógenas, insuficiência renal, hemorragia digestiva alta, neoplasias e alterações glicêmicas.

O tempo de internação na UTI variou de 4 a 96 dias, com mediana de $15(8,0-21,5)$ dias; e a duração da TNE foi de 3 a 91 dias, mediana de 11 (7,0-20,5) dias.

Do total de pacientes estudados, 63,9\% ( $n=94)$ já estavam em uso da TNE no momento da admissão na UTI. Os doentes restantes iniciaram a TNE após a admissão e apresentaram uma variação do tempo de início de 1 a 216 horas, com mediana de 20 (13-33) horas. Tanto a duração da TNE quanto o tempo para início da terapia foram associados à inadequação nutricional (Tabela 2).

Em relação à adequação nutricional da $T N E$, destaca-se que $21,1 \%(n=31)$ dos pacientes receberam volume de dieta e quantidade de proteína inferior a $70 \%$ do prescrito e o restante, acima disso (Figura 1). Na Figura 2, está apresentada a quantidade de proteína prescrita e infundida durante a internação no setor.

O valor da proteína $\mathrm{C}$ reativa no momento da admissão na UTI variou de 0,3 a 425,7 mg/L, mediana igual a $68,0(26,5$ $163,5) \mathrm{mg} / \mathrm{L}$ e não foi estatisticamente significativo quando associado à baixa infusão de volume $(p=0,432)$.

Dentre os fatores que interromperam a administração da TNE, destacaram-se: jejum para procedimentos $(n=55$; $37,4 \%)$ e exames $(n=39 ; 26,5 \%)$ e vômito $(n=31 ; 21,8 \%)$. $\mathrm{Na}$ análise, o volume inadequado associou-se à distensão abdominal e ao vômito (Tabela 3).
Tabela 1 - Dados demográficos e características clínicas ${ }^{a}$. Hospital Nossa Senhora da Conceição, Tubarão (SC), ( $n=147)$.

\begin{tabular}{lc}
\hline Variável & Valor \\
\hline Gênero & \\
Masculino & $68(46,3)$ \\
Feminino & $79(53,7)$ \\
IMC & $27,24 \pm 5,98$ \\
Idade (anos) & $71,0(62,0-79,5)$ \\
\hline Diagnóstico na admissão da UTI & \\
Sistema respiratório & $42(28,6)$ \\
Sepse & $39(26,5)$ \\
Sistema neurológico & $37(25,2)$ \\
Sistema cardiovascular & $19(12,9)$ \\
Outros & $10(6,8)$ \\
\hline
\end{tabular}

a Valores apresentados como número (\%), mediana (p25-p75) ou média \pm DP IMC = índice de massa corporal; UTI = unidade de terapia intensiva.

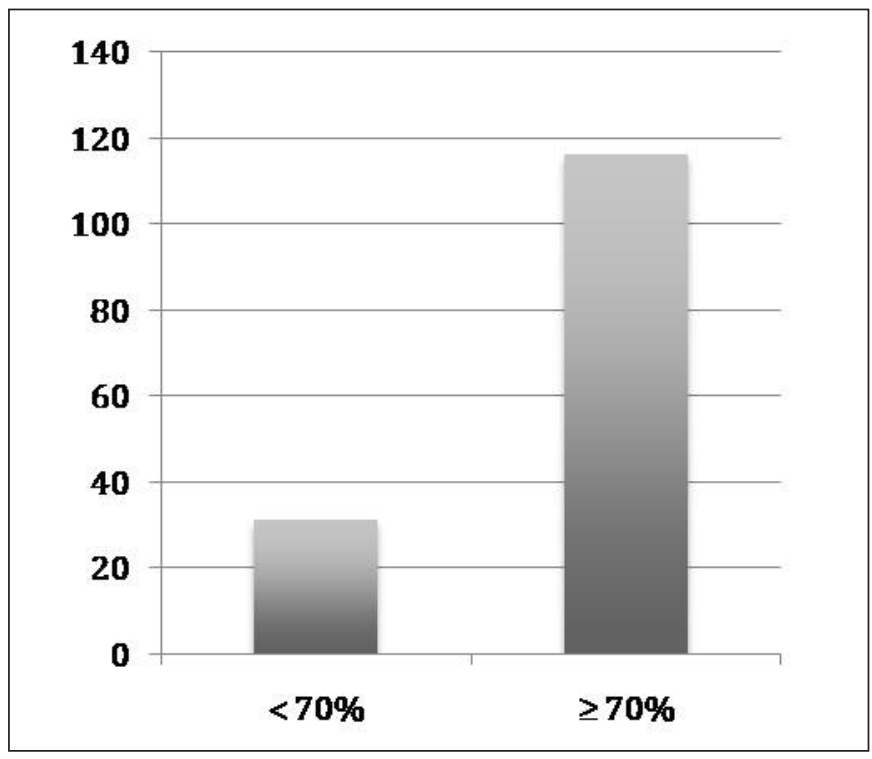

Figura 1 - Adequação nutricional.

Tabela 2 - Análise da inadequação nutricional conforme as variáveis. Hospital Nossa Senhora da Conceição, Tubarão (SC).

\begin{tabular}{|c|c|c|c|}
\hline Variáveis & OR & IC (95\%) & Valor $p$ \\
\hline \multicolumn{4}{|l|}{ Inadequação de volume } \\
\hline Idade (anos) & 0,997 & $0,969-1,025$ & 0,812 \\
\hline IMC & 0,970 & $0,904-1,042$ & 0,406 \\
\hline Tempo para início da TNE (horas) & 1,014 & $1,000-1,028$ & 0,046 \\
\hline Tempo de UTI (dias) & 0,963 & $0,926-1,001$ & 0,059 \\
\hline Tempo de TNE (dias) & 0,939 & $0,891-0,989$ & 0,017 \\
\hline Idade (anos) & 0,991 & $0,965-1,019$ & 0,534 \\
\hline IMC & 0,991 & $0,926-1,060$ & 0,797 \\
\hline Tempo para início da TNE (horas) & 1,015 & $1,001-1,029$ & 0,038 \\
\hline Tempo de UTI (dias) & 0,962 & $0,925-1,001$ & 0,055 \\
\hline Tempo de TNE (dias) & 0,935 & $0,886-0,987$ & 0,015 \\
\hline
\end{tabular}

IMC = índice de massa corporal; PCR = proteína C-reativa; TNE = terapia de nutrição enteral; UTI = unidade de terapia intensiva. 


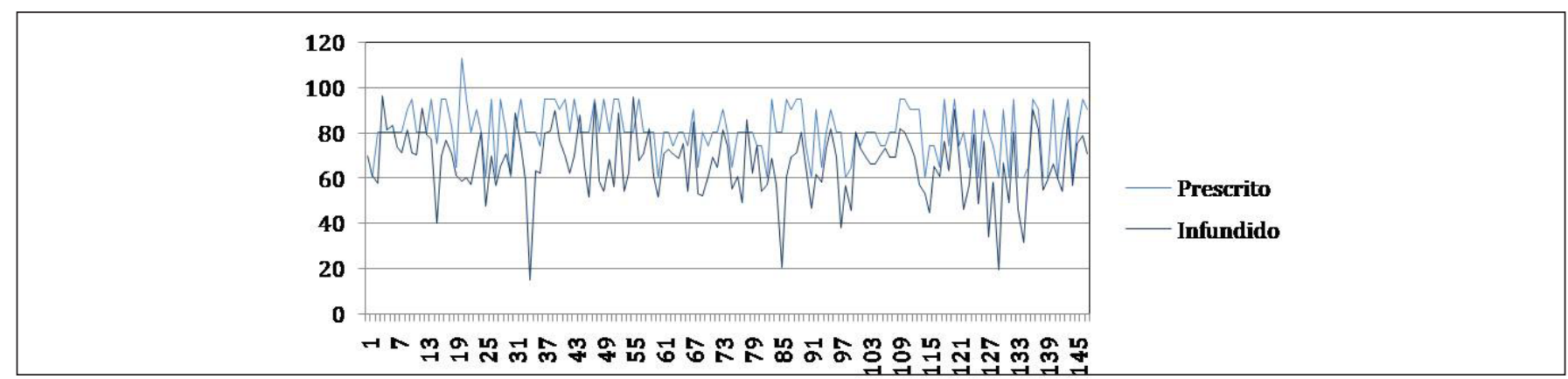

Figura 2 - Proteina prescrita e infundida.

Tabela 3 - Distribuição na amostra, Razão de Chance/Odds Ratio (OR) e Intervalo de Confiança (IC) da inadequação nutricional conforme as variáveis de interrupção da dieta. Hospital Nossa Senhora da Conceição, Tubarão (SC), (n=147).

\begin{tabular}{|c|c|c|c|c|c|c|}
\hline \multirow[b]{3}{*}{ Variáveis } & \multirow{2}{*}{\multicolumn{2}{|c|}{$\begin{array}{c}\text { Distribuição na } \\
\text { Amostra }\end{array}$}} & \multicolumn{4}{|c|}{ Inadequação de volume } \\
\hline & & & \multicolumn{2}{|c|}{ Prevalência } & \multirow{2}{*}{ OR (IC95\%) } & \multirow{2}{*}{ Valor $\mathrm{p}$} \\
\hline & $n$ & (\%) & $\mathrm{n}$ & (\%) & & \\
\hline Sim & 32 & 21,80 & 12 & 37,50 & $3,03(1,27-7,22)$ & \\
\hline Não & 115 & 78,20 & 19 & 16,50 & 1,00 & \\
\hline Jejum para procedimentos & & & & & & 0,867 \\
\hline Não & 92 & 62,60 & 19 & 20,70 & 1,00 & \\
\hline Jejum para exames & & & & & & 0,576 \\
\hline Sim & 39 & 26,50 & 7 & 17,90 & $1,30(0,51-3,32)$ & \\
\hline Não & 108 & 73,50 & 24 & 22,20 & 1,00 & \\
\hline Diarreia & & & & & & 0,808 \\
\hline Sim & 5 & 3,40 & - & - & - & \\
\hline Não & 142 & 96,60 & 31 & 21,10 & 1,00 & \\
\hline Distensão Abdominal & & & & & & 0,009 \\
\hline Sim & 11 & 7,50 & 6 & 54,50 & $5,32(1,50-18,85)$ & \\
\hline Não & 136 & 92,50 & 25 & 18,40 & 1,00 & \\
\hline Saída/Obstrução da sonda & & & & & & 0,346 \\
\hline Sim & 7 & 4,80 & - & - & - & \\
\hline Não & 140 & 95,20 & 31 & 22,10 & 1,00 & \\
\hline Hemodiálise & & & & & & 0,343 \\
\hline Sim & 6 & 4,10 & - & - & - & \\
\hline Não & 133 & 90,50 & 27 & 20,30 & 1,00 & \\
\hline Atraso relacionado ao raio $X$ & & & & & & 0,118 \\
\hline Sim & 18 & 12,20 & 1 & 5,60 & $0,19(0,25-1,52)$ & \\
\hline Não & 129 & 87,80 & 30 & 23,30 & 1,00 & \\
\hline Outros & & & & & & 0,063 \\
\hline Sim & 22 & 15,00 & 8 & 36,40 & $2,53(0,95-6,74)$ & \\
\hline Não & 125 & 85,00 & 23 & 18,40 & 1,00 & \\
\hline
\end{tabular}




\section{DISCUSSÃO}

No presente estudo, a mediana da idade encontrada foi superior à relatada por alguns autores ${ }^{3,11}$. Pesquisa realizada nos Estados Unidos revelou que a maior parte das internações por pneumonia na UTI foi de idosos, principalmente, com mais de 85 anos. Isso acontece porque quanto maior a faixa etária e a presença de comorbidades, maior o risco de admissão dessa população no setor ${ }^{12}$.

A média do IMC foi $27,24 \pm 5,98$, contrapondo-se aos menores valores de IMC $\left(24,3 \pm 18,3 \mathrm{~kg} / \mathrm{m}^{2}\right)$ e idade $\left(51,7 \pm 18,6\right.$ anos) descritos por Santos e Viana ${ }^{13}$, em São Luís, no Maranhão. Porém, na instituição estudada, houve predomínio da população idosa e sabe-se que o processo de envelhecimento é associado a diversas alterações fisiológicas, como acúmulo de tecido adiposo e redução da estatura, que podem ter importantes implicações sobre o estado nutricional e aumento do $\mathrm{IMC}^{14}$.

A mediana do tempo para início da TNE dos pacientes avaliados está de acordo com o proposto pela diretriz americana de nutrição enteral no paciente crítico ${ }^{5}$. Em consonância com esses resultados, Ribeiro et al. ${ }^{15}$ encontraram mediana de $23(14,5-32,0)$ horas, semelhante aos dados do presente estudo. Já outros estudos ${ }^{11,16}$, apesar de apresentarem valores ainda de acordo com o padronizado, tiveram um tempo acima do observado na presente pesquisa. É preconizado o início precoce (24 a 48h) da TNE, por sua associação com melhor resposta nutricional e clínica ${ }^{5}$.

Nesta pesquisa, o tempo mediano de internação na UTI foi de $15(8,0-21,5)$ dias, similar ao tempo de 17,1 (8,0$22,0)$ dias de um estudo realizado em Saitama, no Japão ${ }^{17}$. Além disso, o tempo total de TNE, em dias, também foi ao encontro de uma pesquisa semelhante ${ }^{3}$. Conforme a análise realizada, o tempo prolongado de nutrição enteral foi associado a adequação calórico proteica. Sugere-se, portanto, que quanto mais dias em TNE, maior a quantidade total de dieta infundida.

A oferta não satisfatória de nutrição continua a ser um problema em pacientes críticos. Dados de uma pesquisa realizada em Pelotas - RS demonstram que 34,4\% dos pacientes receberam menos de $70 \%$ da dieta prescrita, valor superior ao encontrado no atual estudo $(21,1 \%)^{18}$. Pesquisa recente propõe administrar a dieta com base no volume total que deverá ser ofertado em $24 \mathrm{~h}$ e alterar a velocidade de infusão conforme as interrupções do dia, a fim de maximizar a oferta nutricional ${ }^{19}$.

No estudo de Ribeiro et al. ${ }^{15}$, foi encontrada correlação negativa da PCR com o volume administrado. Sugerindo, portanto, que as alterações metabólicas da inflamação sistêmica possam resultar em menor tolerância à nutrição enteral, interferindo na oferta alimentar. Por esse motivo, era esperada a associação significativa entre o aumento da PCR e a inadequação calórico proteica. Entretanto, essa relação não foi comprovada nesta pesquisa.

A oferta de nutrição para pacientes críticos pode ser dificultada por diversas barreiras, o que contribui para um déficit significativo de proteína e caloria durante a internação na UTI. Neste estudo, foram identificadas 248 interrupções, destas, $25,8 \%$ devido a sintomas gastrointestinais, seguido de $22,17 \%$ e $15,72 \%$ para realização de procedimentos e exames, respectivamente. Um estudo indiano também demonstrou que cerca de um quarto $(24,03 \%)$ das interrupções foram secundárias a disfunções gastrointestinais ${ }^{20}$. A alta frequência desses sintomas pode ser explicada pela grande quantidade de medicamentos que alteram a função normal do trato alimentar, como opioides, além do predomínio do decúbito dorsal na maioria dos pacientes, o que favorece vômitos e regurgitações. Tais fatores podem ser controlados por meio de medidas posturais, quando possível, e uso adequado de procinéticos e antieméticos ${ }^{21}$.

Dentre os sintomas gastrointestinais, apenas o vômito e a distensão abdominal foram estatisticamente significantes quando associados à inadequação nutricional. Em ambas as situações, o volume de dieta ofertado é reduzido até que haja resolução do quadro e possa se reestabelecer a infusão normal. Isso contribui para uma oferta inadequada durante um período. Os dados de Santana et al. ${ }^{3}$ corroboram com os achados deste estudo e demonstram associação entre inadequação nutricional e vômitos, no entanto, a maior prevalência foi de resíduo gástrico aumentado, assim como na pesquisa de Rocha et al. ${ }^{22}$. Todavia, esse fator não foi encontrado no atual estudo, porque a aspiração nasogástrica não é feita de rotina no serviço.

Outras situações prováveis são problemas relacionados ao CNE. Na UTI estudada, houve demora para troca da via, visto que esta não era trocada imediatamente após a solicitação e atraso na liberação da dieta após o raio-X, - tanto delonga para realização do exame, quanto para avaliação da imagem pelo médico, além disso, obstrução e perda do cateter. Esses motivos podem ser minimizados com protocolos que determinem o tempo de jejum para exames de imagem, cuidados especiais pela enfermagem e fixação adequada da sonda ${ }^{21}$. As situações citadas também foram encontradas por um estudo realizado no Hospital das Clínicas de Goiás ${ }^{3}$ e em outro no Maranhão22.

Assim como na presente pesquisa, vários estudos demonstram o jejum para procedimentos como barreira à oferta nutricional mais encontrada $3,20,22$. A despeito da alta frequência do fator citado (37,4\% dos pacientes), cabe especificar que, no presente estudo, a causa principal foi traqueostomia, assim como na pesquisa de McCall et al. ${ }^{16}$, que teve predomínio do manuseio de vias aéreas. A elevada prevalência se justifica, pois a realização dessa intervenção 
depende de vários fatores, como disponibilidade do centro cirúrgico e estabilidade clínica do paciente. Uma campanha da Sociedade Brasileira de Nutrição Parenteral e Enteral ${ }^{21}$ sugere jejum de apenas 1 hora antes desse procedimento, a fim de reduzir tempo excessivo de interrupção da dieta durante a internação hospitalar. Em contrapartida, no estudo de Uozumi et al. ${ }^{17}$, apenas $3 \%$ dos pacientes foram interrompidos por esse motivo.

Outro fator descrito, porém em menor frequência, foi a interrupção para hemodiálise em 4,1\% dos pacientes. Esse dado foi encontrado em um único estudo, com prevalência de $13 \%{ }^{17}$. Essa barreira foi encontrada, pois, no hospital estudado, os pacientes mais graves são admitidos na UTI para realizar esse procedimento em uma sala especial. Uma forma de melhorar a adequação nutricional é interromper a dieta imediatamente antes do início da sessão e reiniciá-la logo após.

Para alcançar o objetivo de maximizar a adequação calórico proteica, protocolos de nutrição enteral deveriam focar em reduzir o tempo das interrupções e, quando isso não for possível, criar estratégias para compensá-las ${ }^{17,19}$. Deve-se levar em consideração que o prejuízo nutricional pode não ser recuperado no mesmo dia. Artigos recentes propõem algumas soluções às principais barreiras evitáveis ${ }^{5,19,21}$. Por exemplo, classificar os doentes em escores - a fim de identificar aquele que terá maior benefício com a terapia - e definir o tempo mínimo para parar a TNE antes de cada procedimento. Além disso, garantir integração multidisciplinar para o manejo da dieta, com nutricionistas, médicos e enfermeiros, para fornecê-la conforme o recomendado pelas diretrizes e protocolo de cada UTI.

A principal limitação do estudo foi relacionada ao preenchimento incompleto de informações referentes à administração da dieta enteral no prontuário do paciente. No entanto, os dados presentes permitem concluir que os pacientes internados na UTI recebem quantidade menor de dieta que o prescrito pela nutricionista e sugerem algumas causas mais relacionadas à inadequação de volume, como episódios de vômito e distensão abdominal. Vale ressaltar que, as associações encontradas são apenas geradoras de hipóteses e não podem comprovar risco entre as variáveis analisadas.

\section{CONCLUSÃO}

Os pacientes estudados foram predominantemente idosos, do sexo feminino e admitidos na UTI principalmente por doenças respiratórias. A introdução da TNE foi precoce, conforme o preconizado pelas diretrizes.

Em relação à adequação nutricional, 21,1\% dos pacientes receberam volume de dieta e quantidade de proteína inferior a $70 \%$ do prescrito e o restante, acima disso.
Os fatores que interferiram na oferta nutricional foram semelhantes aos encontrados na literatura, com predomínio do jejum para procedimentos, exames e disfunções gastrointestinais. Esses motivos podem aumentar a incidência de desnutrição em pacientes críticos. Dessa maneira, os achados podem ajudar a implementar protocolos na UTI, para otimizar a oferta nutricional durante o período de internação.

\section{REFERÊNCIAS}

1. Jensen GL, Wheeler D. A new approach to defining and diagnosing malnutrition in adult critical illness. Curr Opin Crit Care. 2012;18(2):206-11.

2. Teixeira ACC, Caruso L, Soriano FG. Terapia nutricional enteral em unidade de terapia intensiva: infusão versus necessidades. Rev Bras Ter Intensiva. 2006;18(4):331-7.

3. Santana MMA, Vieira LL, Dias DAM, Braga CC, Costa RM. Inadequação calórica e proteica e fatores associados em pacientes graves. Rev Nutr. 2016;29(5):645-54.

4. McClave SA, Martindale RG, Rice TW, Heyland DK. Feeding the critically ill patient. Crit Care Med. 2014;42(12):2600-10.

5. McClave SA, Taylor BE, Martindale RG, Warren MM, Johnson DR, Braunschweig C, et al. American Society for Parenteral and Enteral Nutrition and Society of Critical Care Medicine. Guidelines for the provision and assessment of nutrition support therapy in the adult critically ill patient: Society of Critical Care Medicine (SCCM) and American Society for Parenteral and Enteral Nutrition (A.S.P.E.N.). JPEN J Parenter Enteral Nutr. 2016;40(2):159-211.

6. Desai SV, McClave SA, Rice TW. Nutrition in the ICU: an evidence-based approach. Chest. 2014;145(5):1148-57.

7. Elke G, Van Zanten AR, Lemieux M, McCall M, Jeejeebhoy KN, Kott M, et al. Enteral versus parenteral nutrition in critically ill patients: an updated systematic review and meta-analysis of randomized controlled trials. Crit Care Med. 2016;20(1):117.

8. Martins JR, Shiroma GM, Horie LM, Logullo L, Silva MLT, Waitzberg DL. Factors leading to discrepancies between prescription and intake of enteral nutrition therapy in hospitalized patients. Nutrition. 2012;28(9):864-7.

9. Sant'Ana IES, Mendonça SS, Marshall NG. Adequação energético-proteica e fatores determinantes na oferta adequada de nutrição enteral em pacientes críticos. Com Ciências Saúde. 2013;22(4):47-56.

10. Arabi YM, Aldawood AS, Haddad SH, Al-Dorzi HM, Tamim HM, Jones G, et al. Permissive underfeeding or standard enteral feeding in critically ill adults. N Engl J Med. 2015;372(25):2398-408.

11. Kozeniecki M, McAndrew N, Patel JJ. Process-related barriers to optimizing enteral nutrition in a tertiary medical intensive care unit. Nutr Clin Pract. 2016;31(1):80-5.

12. Storms AD, Chen J, Jackson LA, Nordin JD, Naleway AL, Glanz $\mathrm{JM}$, et al. Rates and risk factors associated with hospitalization for pneumonia with ICU admission among adults. BMC Pulm Med. 2017;17(1):208.

13. Santos FA, Viana KDAL. Avaliação do estado nutricional e da terapêutica dietética de pacientes internados em uma unidade de terapia intensiva. Rev Pesq Saúde. 2016;17(1):42-6.

14. Lipschitz DA. Screening for nutritional status in the elderly. Prim Care. 1994;21(1):55-67.

15. Ribeiro LMK, Oliveira Filho RS, Caruso L, Lima PA, Damasceno NRT, Soriano FG. Adequação dos balanços energético e proteico na nutrição por via enteral em terapia intensiva: quais são os fatores limitantes? Rev Bras Ter Intensiva. 2014;26(2):155-62.

16. McCall ME,AdamoA, Latko K, RiederAK, Durand N, Nathanson T. Maximizing nutrition support practice and measuring 
adherence to nutrition support guidelines in a Canadian tertiary care ICU. J Intensive Care Med. 2018;33(3):209-17.

17. Uozumi M, Sanui M, Komuro T, Iizuka Y, Kamio T, Koyama H, et al. Interruption of enteral nutrition in the intensive care unit: a single-center survey. J Intensive Care. 2017; 5:52.

18. Gonçalves CV, Borges LR, Orlandi SP, Bertacco RTA. Monitoramento da terapia nutricional enteral em unidade de terapia intensiva: adequação calórico-proteica e sobrevida. BRASPEN J. 2017;32(4):341-6.

19. Kozeniecki M, Pitts H, Patel JJ. Barriers and solutions to delivery of intensive care unit nutrition therapy. Nutr Clin Pract. 2018;33(1):8-15.
20. Ramaakrishnan N, DaphneeDK, Ranganathan L, Bhuvaneshwari S. Critical care $24 \times 7$ : but, why is critical nutrition interrupted? Indian J Crit Care Med. 2014;18(3):144-8.

21. Toledo DO, Piovacari SMF, Horie LM, Matos LBN, Castro MG, Ceniccola GD et al. Campanha "diga não à desnutrição": 11 passos importantes para combater a desnutrição hospitalar. BRASPEN J. 2018; 33(1):86-100.

22. Rocha AJSC, Oliveira ATV, Cabral NAL, Gomes RS, Guimarães TA, Rodrigues WB, et al. Causas de interrupção de nutrição enteral em unidades de terapia intensiva. Rev Pesq Saúde. 2017; 18(1):49-53.

Local de realização do estudo: Universidade do Sul de Santa Catarina (UNISUL), Tubarão, SC, Brasil.

Conflito de interesse: Os autores declaram não haver. 